\title{
Current Topics in the Stochastic Theory of Radiation
}

\author{
Francis J. Zucker

\begin{abstract}
Contribution From Air Force Cambridge Research Laboratories, Office of Aerospace Research, Bedford, Mass.
\end{abstract}

(Received March 31, 1964)

\begin{abstract}
The stochastic properties of fluctuating electromagnetic fields are defined in terms of the joint moments of the probability distribution. Their physical interpretations (coherence, higher order correlations) are briefly described, and the connection is indicated between the complete set of correlations and the quantum theory of radiation.
\end{abstract}

\section{Introduction}

This paper deals with the stochastic theory of radiation. It is the electromagnetic field itself which fluctuates here, not the medium: we are simply concerned with an extension to the space domain of the usual communication-theoretical treatment of signals fluctuating in time.

As usual in a stochastic theory, we must define the variable and its ensemble, and describe a sequence of joint probability densities that specify the statistical properties in greater and greater detail. Let $V(x, t)$ represent a voltage or electric field strength (scalar for the time being) at point $x$ and time $t$. Since the bandwidth of a physically realizable radiation field is never zero, $V$ cannot be periodic in time but must fluctuate. Let the ensemble be a set of wavefields produced by one and the same source at different times. If $p_{1}\left(V_{1}\right) d V_{1}$ is the probability that at the space-time point $\left(x_{1}, t_{1}\right), V$ will have a value that lies between $V_{1}$ and $V_{1}+d V_{1}$, then the successive orders of joint probability densities are defined as follows: $p_{2}\left(V_{1}, V_{2}\right) d V_{1} d V_{2}$ is the joint probability that at the space-time point $\left(x_{1}, t_{1}\right), V$ will lie within $d V_{1}$ and at $\left(x_{2}, t_{2}\right)$, within $d V_{2}$; $p_{3}\left(V_{1}, V_{2}, V_{3}\right) d V_{1} d V_{2} d V_{3}$ refers analogously to three space-time points, etc.

Certain weighted integrals of the joint probabilities often turn out to have direct physical significance: these are the joint moments, defined by the ensemble average

$$
\begin{aligned}
& \Gamma^{(i)} \equiv \overline{V_{1} V_{2} \ldots V_{i}} \\
& \equiv \int_{-\infty}^{\infty} \ldots \int_{-\infty}^{\infty} V_{1} V_{2} \ldots V_{i} p_{i}\left(V_{1}, V_{2},\right. \\
& \left.\ldots V_{i}\right) d V_{1} d V_{2} \ldots d V_{i}
\end{aligned}
$$

or equivalently, since we shall assume quasiergodicity, by the time average

$$
\Gamma^{(i)} \equiv \lim _{T \rightarrow \infty} \frac{1}{2 T} \int_{-T}^{T} V_{1} V_{2} \ldots V_{i} d t
$$

which is usually abbreviated as $\Gamma^{(i)} \equiv\left\langle V_{1} V_{2} \ldots V_{i}\right\rangle$. If the fields are stationary in time (and for convenience we shall here restrict ourselves to these), the products $V_{1}\left(x_{1}, t_{1}\right) V_{2}\left(x_{2}, t_{2}\right) V_{3}\left(x_{3}, t_{3}\right)$. . . become $V_{1}\left(x_{1}, t\right) V_{2}\left(x_{2}, t+\tau\right) V_{3}\left(x_{3}, t+\tau^{\prime}\right) \ldots$, and the secondorder joint moment, for example, is then explicitly written as

$$
\Gamma^{(2)} \equiv\left\langle V_{1}(t) V_{2}(t+\tau)\right\rangle
$$

(omitting the $x_{1}$ and $x_{2}$ ), which is recognized as the cross correlation of $V_{1}$ and $V_{2}$. In the case of quasi-ergodicity and stationarity, therefore, the terms "joint moment" and "correlation" are interchangeable.

It is often convenient (and in the context of the quantum theory of radiation, necessary) to work with the complex analytic signal $V$ rather than the real signal $V$, the imaginary part of $\underset{\sim}{V}$ being defined as the Hilbert transform of $V$ (actually, since $V$ is a random function and therefore nonsquareintegrable, its analytic continuation involves a detour via a truncated $V_{T}$ ). In terms of the analytic signal, the second-order correlation, for example, is

$$
\Gamma^{(2)} \equiv\left\langle{\underset{\sim}{1}}_{1}^{*}(t) \underset{\sim}{V_{2}}(t+\tau)\right\rangle \equiv \Gamma_{12}(\tau) .
$$

What is the physical significance of the several orders of correlation? The first order is just the time-average voltage (electric or magnetic) field at a point, which is usually zero. The second order, $\Gamma_{12}(\tau)$, traditionally called the coherence function, enters in the mathematical description of all interference and diffraction effects, and of instruments based on these (e.g., the ordinary radio interferometer). Referred to a single point, $\Gamma_{12}(\tau)$ becomes $\Gamma_{11}(\tau) \equiv\left\langle V_{1}^{*}(t) V_{1}(t+\tau)\right\rangle$, the autocorrelation; in terms of $\tilde{I}(t)$, the fluctuating "intensity" (or, except for an admittance factor, the electromagnetic power flow), $\Gamma_{11}(\tau)=\left\langle I_{1}(t)\right\rangle=I_{1}$, the mean (or "optical") intensity at the point. The third order, I heard recently, is being examined at present in connection with the correlations between incident, reflected, and transmitted rays in nonlinear interactions between light and matter. A degenerate form of the fourth-order correlation, applied to two rather than four points: 


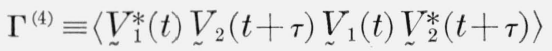

$$
\begin{aligned}
& =\left\langle I_{1}(t) I_{2}(t+\tau)\right\rangle \text {, }
\end{aligned}
$$

the cross correlation between the intensity fluctuations at two points, underlies all intensity interferometry (Hanbury Brown-Twiss effect, etc.) and two-point photoelectron coincidence counting. The fifth order seems to have no application. The sixth, in a degenerate form applying to only three points, appears in H. Gamo's triple-correlator interferometers which, for certain specialized measurements (for example, the spectrum of gas discharge tubes) offer important advantages over ordinary amplitude and intensity interferometers. Orders higher than the sixth have not so far been found physically significant, but the set of all orders taken as a whole, i.e., the complete stochastic description of the field, turns out to be highly significant in the quantum theory of radiation. We shall return to this point after examining the second- and fourth-order correlations in somewhat more detail.

\section{Second-Order Correlations}

In figure 1, a plane, circular source of diameter $2 \rho$ illuminates slits 1 and 2 in a screen; we will show that in the case of quasi-monochromatic radiation, the intensity $I(P)$ at point $P$ depends on the second-order correlation, the coherence function $\Gamma_{12}(\tau)$. Let $\underset{\sim}{V_{1}}(\omega)$ be a spectral amplitude component of the voltage ${\underset{\sim}{1}}_{1}(t)$ at slit 1 , and note that the corresponding power spectral line of $I_{1}(t)$ is $i_{1}(\omega)=V_{1}(\omega) * V_{1}(\omega)$. The voltages at $\mathrm{P}$ superimpose: $V(P, \omega)=a_{1} \tilde{V}_{1}(\omega) \exp \left(i k r_{1}\right)+$ $a_{2} V_{2}(\omega) \exp \left(i k r_{2}\right)$ (where the $a$ 's represent the individual slit patterns and the $1 / r^{2}$ decay), and therefore

$$
\begin{aligned}
& i(P, \omega)=\langle\underset{\sim}{V}(P, \omega) \underset{\sim}{V}(P, \omega)\rangle \\
&=\left|a_{1}\right|{ }^{2} i_{1}(\omega)+\left|a_{2}\right|^{2} i_{2}(\omega) \\
&+2\left|a_{1}\right|\left|a_{2}\right| \operatorname{Re}\left\{\left\langle\underset{\sim}{V_{1}}(\omega) \underset{\sim}{\left.\left.V_{2}^{*}(\omega)\right\rangle e^{-i \omega \tau}\right\},}\right.\right.
\end{aligned}
$$

where $\omega \tau$ is the phase difference corresponding to the path-length difference $c \tau$ in ifgure 1. (All mathematical details involving the truncated functions have been omitted.) The total $I(P)$ is obtained by integrating $i(P, \omega)$ over the bandwidth $\Delta \omega<<\bar{\omega}$ (the

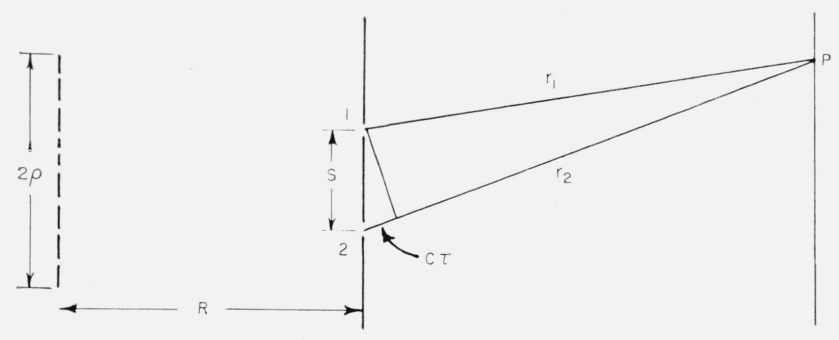

Figure 1. Two-slit interference. mean frequency); noting that the last term on the right-hand side then represents the Fourier transform of the mutual power spectrum between slits 1 and 2 , which is well known (Wiener-Khinchine theorem) to be the cross correlation function, we obtain

$$
I(P)=\left|a_{1}\right|^{2} I_{1}+\left|a_{2}\right|^{2} I_{2}+2\left|a_{1}\right|\left|a_{2}\right|\left|\Gamma_{12}(0)\right| \cos \bar{\omega} \tau,
$$

in which the last two factors could also be written as $\operatorname{Re} \Gamma_{12}(\tau)$. (At radio frequencies, amplitude interferometers are used in lieu of the optical arrangement of figure 1: a phase shifter produces the path length difference, and either, as in figure $2 \mathrm{a}$, a detector registers $I(P)$ or else, as in figure $2 \mathrm{~b}$, a correlator consisting of multiplier and integrator reads out $\mathrm{I}_{12}(\tau)$ directly.) It can be easily shown that $\left|\Gamma_{12}(0)\right|$ must lie between $\sqrt{I_{1} I_{2}}$ and 0 . If we choose $I_{1}=I_{2}=I$, then $I(P)=2|a|^{2} I(1+\cos \bar{\omega} \tau)$ at the upper bound and $I(P)=2 a^{2} I$ at the lower, as illustrated in figure $3 \mathrm{a}$ (deep nulls, "complete coherence") and figure 3d (no nulls, "complete incoherence"), respectively; the dashed curve in (a) and solid curve in (d) trace the individual slit pattern contained in the coefficient $a$. Cases (b), (c), and (e) are intermediate ("partial coherence"): for small $\tau$ (near the center), the fringe contrast is good, but gradually it "washes out." The approximate path-length difference at which the fringes disappear for visual observation is called the "coherence length" $c \tau_{c}$, where the "coherence time" $\tau_{c} \cong 2 \pi / \Delta \omega$; the coherence length is about $0.1 \mu \mathrm{m}$ for white light, 3 yards for a very narrow spectral line, 200 miles for a good laser, and 2,000 miles for a wellstabilized klystron.

The central fringe contrast $\Gamma_{12}(0)$, termed the "spatial coherence," can be measured in the Michelson two-beam interferometer, figure 4a. If the source is incoherent (e.g., a star), the Fourier transform of $\Gamma_{12}(0)$ plotted against increasing separation between incident beams traces out the intensity distribution across the source (and thus also measures its diam-



FIgure 2. Two microwave amplitude interferometers: (a) with phase shifter and detector D, (b) with multiplier M and integrator $\mathrm{I}$. 


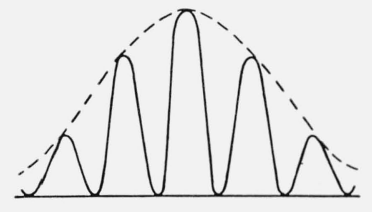

(a)

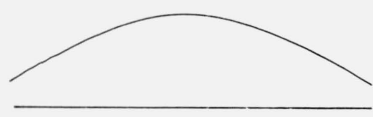

(a)

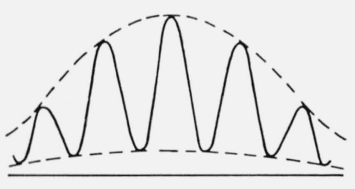

(b)

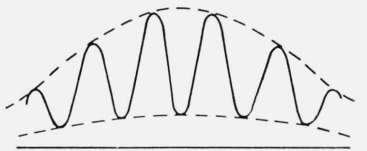

(c)

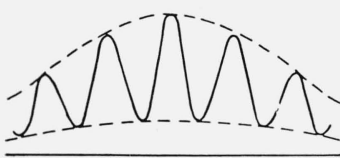

(c)

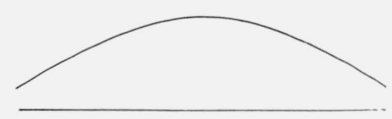

(f)

Figure 3. Two-slit interference patterns.

eter). The autocorrelation $\Gamma_{11}(\tau)$, termed the "temporal coherence," can be measured in the Michelson split-beam interferometer, igure $4 \mathrm{~b}$. The Fourier transform of $\Gamma_{11}(\tau)$ plotted against increasing pathlength difference between mirrors 1 and 2 traces out the power spectrum of the source.

The proof of these relations is best obtained from the double wave equation

$$
\left(\nabla_{n}^{2}-\frac{1}{c^{2}} \frac{\partial^{2}}{\partial \tau^{2}}\right) \Gamma_{12}(\tau)=0, \quad n=1,2
$$

which Wolf established for the coherence function; it is a consequence of the scalar wave equation satisfied by the analytic signals themselves. Thus the coherence function changes as light propagates in space: for example, complete spatial incoherence at the surface of a star is transformed into almost complete coherence by the time the light enters the aperture of a telescope (or else no Airy rings would be formed). With the wave equation one can also prove that the interference diagrams in figure 3 depend on the parameter-combination $\bar{\omega} s p / R$ in figure 1: increasing the mean frequency of the incoherent source, or its diameter, or the slit separation, or decreasing the source distance, all produce precisely the sequence of patterns shown in figure 3 . (Note how the fringe contrast reappears in (e) after it has already been washed out in (d), though with less contrast than at its peak in (a), and with a minimum rather than maximum at the center; all of these effects stem from the behavior of the coherence function.)

The vector nature of electromagnetic waves is taken into account by writing $\Gamma^{(2)}$ as a matrix. For polarized light beams, it is sufficient to work with

$$
\left[\Gamma_{12}(\tau)\right] \equiv\left[\begin{array}{ll}
\Gamma_{x x}(\tau) & \Gamma_{x y}(\tau) \\
-\Gamma_{4}(\tau) & \Gamma_{y y}(\tau)
\end{array}\right],
$$
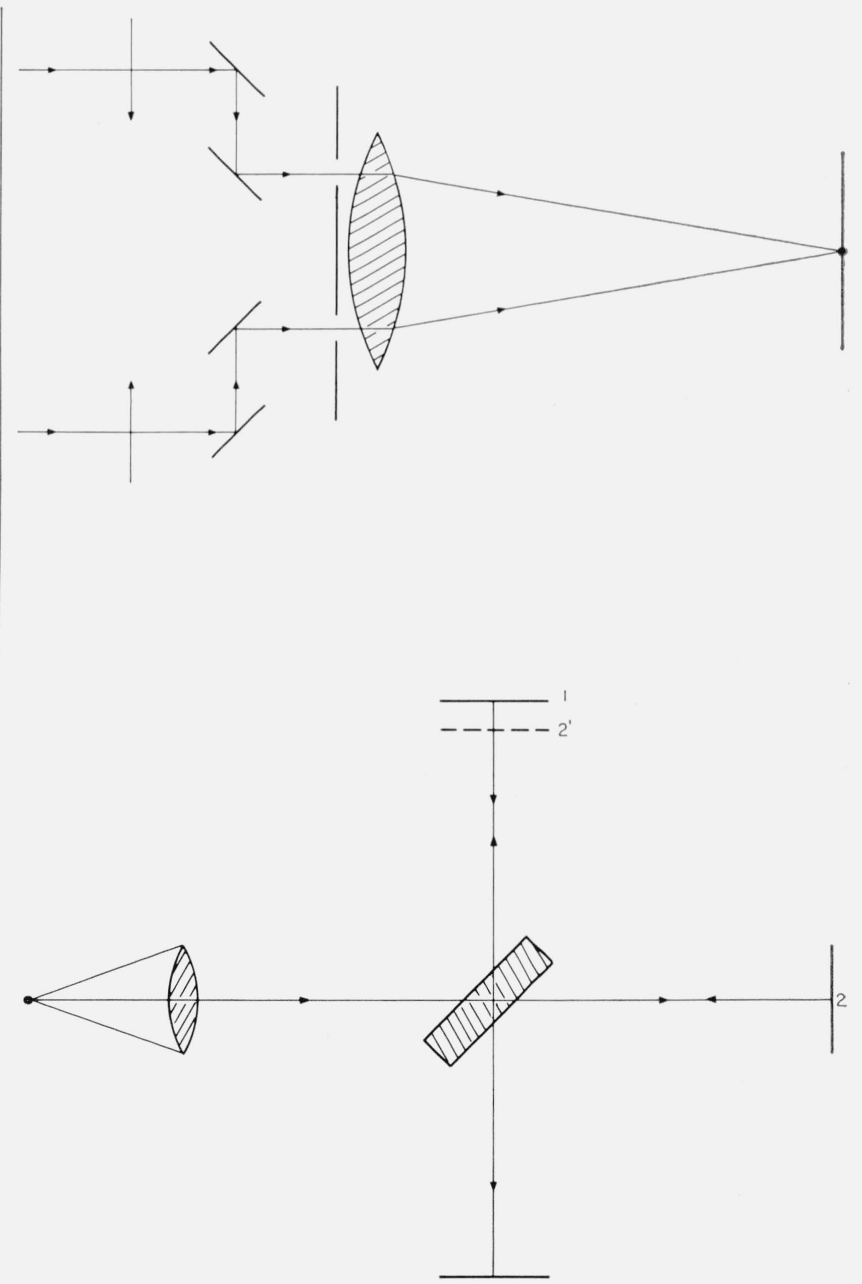

Figure 4. Michelson interferometers: (a) two-beam, (b) splitbeam. 
where the subscripts specify which two transverse $E$ or $H$ field components are being correlated. In the special case $\left[\Gamma_{11}(0)\right]$, these four parameters characterize a quasi-monochromatic beam in the same way as the Stokes vector characterizes a monochromatic beam; the familiar polarization algebra can therefore be developed, including representation on a Poincaré sphere, splitting an arbitrary beam into a fully polarized and a fully unpolarized part, etc.

References to the many applications of the coherence calculus in diffraction, antenna patterns, optical imaging, radio astronomy, periodic and random media, etc., will be found in the last item listed under "Bibliography" at the end of this article.

\section{Fourth-Order Correlations}

Thermal sources consist of independently-radiating atoms; we know from the central limit theorem that all orders of the probability density distributions must then be Gaussian, and that all higher joint moments can be expressed in terms of the second. In particular, the degenerate two-point fourth-order correlation already introduced turns out to be related to the coherence function by

$$
\left\langle I_{1}(t) I_{2}(t+\tau)\right\rangle=I_{1} I_{2}+\left|\Gamma_{12}(\tau)\right|^{2}
$$

if the field is linearly polarized; otherwise, a factor multiplies the last right-hand term, for example 0.5 if the field is unpolarized. It is clear from this expression that two-point intensity correlation yields only the magnitude of the coherence function, but the phase is sometimes recoverable by theoretical arguments, and often recoverable by additional measurements, for example with the three-point interferometer mentioned in the Introduction.

In the Hanbury Brown and Twiss experiment, figure $5,\left|\Gamma_{12}(0)\right|$ is determined by the intensityanalog of the two-beam amplitude interferometer in figure 4a: the light or radio signals now are detected $D$ and amplified $A$ before being correlated in the multiplier-integrator unit. A longer baseline can therefore be used than alinement problems and atmospheric turbulence permit with the Michelson interferometer, but because of energy limitations only a handful of stars have been mapped in this

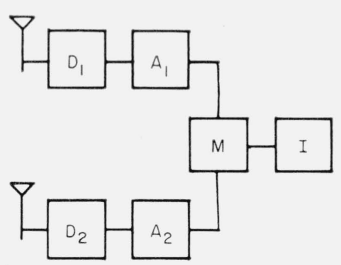

Figure 5. Intensity interferometer. way. The intensity analog of figure $4 \mathrm{~b}$ yields $\left|\Gamma_{11}(\mathrm{~T})\right|$ and therefore the source spectrum (magnitude only unless known to be symmetric).

Of the several other experiments that depend on intensity correlation, I will mention only that of Alford and Gold, in which power spectrum modulations are observed when a beam is recombined after a path-length difference in excess of the coherence length.

The fourth-order correlation of nonthermal sources, such as lasers and klystrons, does not depend on the coherence function; in fact, an ideal single-mode amplitude-stabilized oscillator should produce intensity fluctuations of zero correlation.

In optics, two-point coincidence counting is often used in lieu of intensity correlation (Pound and Rebka experiment, etc.). The equation that establishes a stochastic relationship between the probability distribution of the photoelectrons in time and the intensity fluctuations is

$$
p(t) d t=\alpha I(t) d t,
$$

where $\alpha$ depends on the efficiency of the photodetector. Originally suggested by Purcell on a semiclassical basis applicable to monochromatic fields only, this relation is now known to hold for polychromatic fields within the accuracy of firstorder quantum-mechanical perturbation theory. A straightforward calculation shows that the probability of finding $n$ photons in a time $T$ consists, for thermal radiation, of two terms: a classical particlelike Poisson distribution added to a wavelike Bose-Einstein distribution (not to be confused with the basic Bose-Einstein statistics which underlies all photon distributions). For ideal laser or klystron radiation, the distribution is completely Poisson, i.e., like shot noise - which explains why there can be no two-point intensity correlation in this case.

Returning to thermal radiation, the variance in the number $n_{T}$ of photoelectrons ejected during $T$ follows from the distribution

$$
\overline{\left(\Delta n_{T}\right)^{2}}=\overline{n_{T}}+\alpha^{2} \overline{\left(I_{T} T\right)^{2}},
$$

which again is the sum of a term due to classical particles and one due to classical waves-just like Einstein's celebrated blackbody fluctuation formula, but now valid also for radiation that is not in thermal equilibrium. It can be shown from this expression that the two-point coincidence counts, for $T>>\tau_{c}$ and linear polarization, are given by

$$
\Delta n_{1} \Delta n_{2}=\alpha^{2} \tau_{c} T\left|\Gamma_{12}(0)\right|^{2}
$$

(with a factor 0.5 for unpolarized light). We have thus available a third method for measuring the intensity distribution across incoherent sources; a related procedure yields spectral information.

The fourth-order correlation propagates through space in accordance with a four-fold wave equation. (And it is true in general that an $n$ th-order correlation satisfies an $n$-fold set of wave equations.) 


\section{Connection With the Quantum Theory of Radiation}

It is well known that statistical mechanics, which treats ensembles of classical particles, contains more physics than one might expect from an $n$-body problem in classical mechanics; in particular, it encompasses irreversibility, whereas the equations of classical mechanics are reversible. This enrichment in physical content comes from the assignment of probability distributions to quantities that are sharply defined classically (for example, the velocity of molecules), and from certain auxiliary concepts, especially equipartition and the rules for counting degrees of freedom.

Here, too, we have "enriched" a classical theory with probability distributions; can auxiliary concepts now be added so as to produce a physical theory of larger scope than classical electrodynamics? The answer is affirmative: the stochastic theory of classical radiation we have just sketched can be further developed so as to encompass quantum effects; in fact, recent work by Glauber and Sudarshan shows that, for all linear interactions, the enlarged stochastic theory is isomorphic with the quantum theory of electromagnetic fields.

The basic correspondence is found to be that between the complete sequence $p_{1}, p_{2}, p_{3} \ldots$. of probability distributions with which we introduced the classical ensemble, and the quantum-mechanical density matrix; the classical correlations are then the expectation values of the quantum-mechanical observables. Two auxiliary concepts are implied by this equivalence: First, because the quantum-me- chanical operator representing the electromagnetic field is complex, use of the analytic signal becomes a necessity rather than a convenience. And second, the Hermiticity of the density matrix forces the probability distribution to become negative over certain ranges of the field variables; these turn out to be unobservable because they violate the uncertainty principle.

Could it be that the confrontation of the engineer with areas of knowledge previously reserved to the pure physicist, which occurred during the early 'Fifties in solid-state electronics, and more recently in maser and laser technology, will now repeat itself in the field of radio and optical wave propagation?

\section{Annotated Bibliography}

Beran, M., and G. B. Parrent (1964), Theory of Partial Coherence (Prentice-Hall, Englewood Cliffs, N.J.). Second- and fourth-order theory (except for coincidence counting) are thoroughly described in this book.

Born, M., and E. Wolf (1959), Principles of Opties, ch. 10 (Pergamon Press, London). The standard reference for second-order theorv.

Mandel, L. (1963), Fluctuations of light beams, Progress in Optics, 2, ed. E. Wolf (North-Holland Publishing Co., Amsterdam). Covers fourth-order theorv.

Mandel, L., E. C. G. Sudarshan, and E. Wolf (1964), Stochastic theory of photoelectric detection of light fluctuations, Phys. Rev. 134, to be published. Discusses quantum aspects of fourth-order theory.

Zucker, F. J. (1964), Partially-coherent electromagnetic fields, Radio Sci. J. Res. NBS/USNC-URSI 68D, No. 4, 460-462. Lists detailed references to all of the topics mentioned in Current Topics in the Stochastic Theory of Radiation.

(Paper 68D9-398) 